\title{
Effect of Axial and Radial Velocities on Solids Mass Flow Rate Measurement
}

\author{
Jianyong Zhang*, Bin Zhou**, Shimin Wang** \\ *School of science and Engineering, Teesside University, Middlesbrough, TS1 3BA United Kingdom \\ ** School of Energy and Environment, Southeast University, Nanjing, China
}

\begin{abstract}
In coal powder flow transportation and combustion, powder mass flow rate is a key parameter to be monitored and controlled. Electrostatics is one of the techniques used for such task with its non-intrusive, robust and low cost natures. The passive electrostatic meters measure charge induced on the detecting electrodes. As it is known that the induced signal is not only dependent on the solids mass flow rate, but also affected by solids velocity. However the velocity of particles usually referred to is the axial velocity. In reality, the solids velocity is a vector, its projections in both the radial and tangential directions also need to be investigated. This paper analyzes the dynamic sensitivity of ring-shaped electrostatic sensors using finite element method (FEM), and investigates the influence of the axial and radial velocities on the induced signal on the electrodes.
\end{abstract}

Keywords: electrostatic sensor, spatial sensitivity, velocity

\subsection{INTRODUCTION}

In pneumatically conveyed solids flow, electrification occurs due to friction, collision and separation between particles and pipe wall [1,2]. Many factors, such as particle shape, particle size, coarseness of the inner surface of pipe wall, chemical composition of solids, travel distance, humidity, flow profile and solids velocity affect the amount of charges carried by particles [3]. Although electrostatic charging creates problems for industries [4-7], it has also found various applications [8-11]. It is well known that the electrostatic technique has been employed to measure gas-solids flow [12-15], which is the subject to be discussed in this paper.

The ring-shaped electrostatic sensors can be classified into two types according to probe (electrode) construction, the flush-mounted sensor and the lined sensor. A "flush-mounted" sensor, depicted in Fig.1 has its inner surface exposed to flow and is mounted flush with inner pipe wall [16]. This simple configuration offers high sensitivity to the charge carrying particles. In contrast, there is an insulator such as a ceramic tube inside a "lined sensor", the electrode of such sensor has no direct physical contact with flow. In this paper, the analyses are based on the former.

The axial velocity of charged particles has been measured using the cross correlation method [16] or the spatial filtering method. However the axial velocity provides only the mean velocity of the solids flow along the pipe axis. Consider an individual particle, or a small volume of air-solids mixture, its velocity is actually a vector due to the turbulent motion of particles. The velocity vector can be projected in the axial, radial and tangential directions in a column coordinate reference system. It has long been known that the solids velocity affects the charge induction on the electrode; obviously, it is inadequate to consider axial velocity only. The main purpose of the research presented in this paper is to establish the relationship between the velocity vector and the sensor's output to study the sensing mechanism of the flush-mounted ring-shaped electrostatic sensor for air solids flow measurement. It is suggested that the dynamic sensitivity of the sensor depends upon the amount of the charge carried by particles, on the locations of charged particles, and on the velocities in both radial and axial directions.

\subsection{Simulation based on 3-D FEM}

\subsubsection{Configuration of electrostatic sensor}

Fig.2 (a) shows the configuration of a flush-mounted electrostatic sensor comprised of a short spool piece and a ring probe. The ring probe is surrounded by an insulator layer preventing it from conductive contact with the pipe wall as shown in Fig. 2 (b). The earthed metal pipe wall acts as an electrostatic screen naturally. The micro-column inside the pipe in the above figure represents a charged element, which can be regarded as a group of particles evenly dispersed in this column. The size of the element has to be small enough so that it can be regarded as a charge source surrounded by

* Corresponding author: Tel.: (0044) 1642-342546; Fax: (0044) 1642-342401; E-mail: j.zhang@Tees.ac.uk 
the medium of uniform permittivity.

\subsubsection{Mathematical model}

The purpose of modeling is to find the relationship between the sensor's output voltage and the amount of charge carried by an element, its location and its velocity.

The modelling is based on electrostatic theory, and the magnetic field due to moving charged particles is ignored. The electrostatic equilibrium system is governed by Poisson equations under the given boundary conditions,

$$
\begin{aligned}
& \left(\nabla \quad \underline{\varepsilon} \varepsilon \ll, y, z \nabla u \ll, y, z_{-}=7 D(x, y, z)=-\rho x, y, z\right) \\
& \{u \leftrightarrow, y, z]_{a, y, z \in \in_{p}}=0 \\
& u \ll, y, z, t]_{\varangle, y, z \in \in_{e}}=\text { const } \\
& E_{\infty}=0
\end{aligned}
$$

where $u(x, y, z)$ is the potential distribution. $\Gamma_{p}$ represents the inner surface of the grounded pipe with zero potential, $\Gamma_{e}$ is the floated ring probe, which will be virtually earthed if a charge amplifier is connected to the electrode. $\rho(x, y, z)$ is the charge density distribution, $\varepsilon_{0}$ and $\varepsilon(x, y, z)$ are the permittivity in free space and relative permittivity distribution of particulate material respectively. $D(x, y, z)$ is the electric flux distribution, $\left.D(x, y, z)=z_{,} \varepsilon^{\prime} x, y, z\right) E(x, y, z) . E(x, y, z)$ denotes the electric field strength, and $E_{\infty}$ is the electric field strength at the infinite distance point, $E_{\infty}=0$.

According to the electrostatic theory, if the distribution of relative permittivity $\varepsilon(x, y, z)$ and charge density $\rho(x, y, z)$ are known, One and the only one solution exists under the given boundary conditions to Equation (1). Hence, the distributions of $\mathrm{D}(\mathrm{x}, \mathrm{y}, \mathrm{z})$ and $\mathrm{E}(\mathrm{x}, \mathrm{y}, \mathrm{z})$ can be found, and the induced charge on the ring probe are given by,

$$
\left.Q=\int_{s} D(x, y, z) \cdot d s=\varepsilon \int_{s} \varepsilon x, y, z\right) \cdot E(x, y, z) \cdot d s
$$

where $s$ is the surface of the ring probe.

Consider the complexity of gas-solids flow, the analytical solution to Equation (1) can hardly be found. The 3-D finite element analysis method is used to obtain a numerical solution instead.

For 3-D finite element analysis, an alternative equivalent integral form of Equation (1) can be expressed as follows, where $F(u)$ is known as the "energy function".

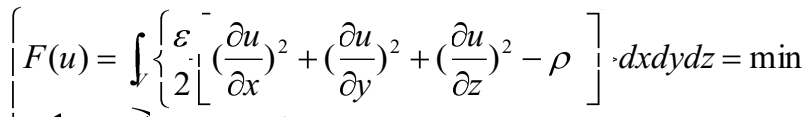

$$
\begin{aligned}
& \{u \ll, y, z]_{\mathbb{*}, y, z \in p}=0 \\
& \{u \llbracket, y, z, t]_{\bullet, y, z \in \in_{e}}=\text { const } \\
& E_{\infty}=0
\end{aligned}
$$

The 'Tetrahedron element' depicted in Fig. 3 is used to discretize the whole field due to its simple coefficient matrix and adaptability to sophisticated field profile.

The relation between the potential anywhere inside the tetrahedron volume $\widetilde{u}(x, y, z)$ and the potentials at its four vertexes, $u_{i}(i=1,2,3,4)$ can be expressed by the interpolation function:

$$
\widetilde{u}(x, y, z)=\sum_{1}^{4} N_{i}^{e} u_{i}
$$

where $N_{i}^{e}$ is the shape function.

After discretization and interpolation are processed, the total energy function $F(u)$ of the volume can be obtained from that of individual element, and then the finite element equations can be constructed in the following way:

Dividing the entire volume of the system into $e_{0}$ elements and $N_{0}$ nodes, the total energy function $F(u)$ can be expressed as 


$$
F(u) \approx F(\tilde{u})=\sum_{e=1}^{e_{0}} F_{e}(\tilde{u})
$$

where $F_{e}(u)$ is the energy function for each tetrahedron element.

The solution to Equation (3) can be found by calculating the extremum of $F(u)$. Equation (6) provides a necessary condition for $F(u)$ to be the extremum.

$$
\frac{\partial F}{\partial u_{i}}=\sum_{e=1}^{e_{0}} \partial F_{\underline{e}}=0 \quad\left(i=, 2, \ldots, N_{0}\right)
$$

The dicretization of equation (3) to each element results in,

$$
\left.\left.F_{e}(u) \approx F_{e}(\tilde{u})=\int\left\{\frac{\varepsilon}{2}\left[\left(\frac{\partial}{\partial}\right)^{2}+\frac{\partial}{\partial}\right)^{2}+\frac{\partial}{\partial}\right)^{2}\right]-,\right\} \mathrm{d} x \mathrm{~d} y \mathrm{~d} z
$$

where $V_{e}$ is the volume of an element.

Substitute $\tilde{u}(x, y, z)$ with $\sum_{i=1}^{4} N_{i}^{e} u_{i}$ based on equation (4), the partial differentiation of $F_{e}(\widetilde{u})$ becomes,

$$
\left.\left.\left[\frac{\partial F_{e}}{\partial u_{i}}\right]=\boldsymbol{K}_{e}^{-}[]_{e}\right]-\boldsymbol{P}_{e}\right]=1 \quad\left(i=, 2, \ldots, n_{0}\right)
$$

where $[K]_{e},[P]_{e},[u]_{e}$ stand for coefficient matrixes of electric field energy, boundary condition and potential for each element.

The coefficient matrix $[K]_{e}$ for each element is symmetrical. For the field divided into $e_{0}$ elements and $N_{0}$ nodes, matrixes $[K]_{e},[P]_{e},[u]_{e}$ can be expanded into $\mathbf{K}_{-}^{-}, \mathbf{F}_{-}^{-},[u]$, the total coefficient matrixes for the whole volume, are:

$$
\mathbf{E}=\sum_{e=1}^{e_{0}} K_{e}^{-}, \mathbf{F}=\sum_{e=1}^{e_{0}} P_{e}^{-}
$$

From the above, the finite element equations can be expressed as

$$
\mathbf{F}=\mathbf{F}
$$

For any given boundary conditions, the solution of equation (10) can be obtained.

The condition of charge equilibrium needs to be satisfied to the best in the numerical simulation, ideally the charge $Q_{e}$ carried by the charged element should be balanced by the sum of $Q_{p}$ and $Q_{r}$, the induced charge on the pipe wall and on the ring probe respectively. That is to say $\left(Q_{p}+Q_{r}\right)$ has the same magnitude as $Q_{e}$, but with opposite polarity. However, restricted by the computation resource, there have to be compromises between the calculation accuracy and the simulation length of the pipe. The length used in this paper is 6 times the internal radius of the pipe, $l=6 R$, which can guarantee the accuracy without putting too much burden on the computation.

Fig. (4) (a), (b) and (c) illustrate meshes on the pipe, ring probe and charged element respectively. Fig.5(a) shows that the local potential distribution is characterized spherically symmetrical with regard to the charged micro-column positioned in the geometrical centre of the pipe; (b) shows the distribution of electrical field strength on the inner pipe wall. The captions on the left top of $(a, b)$ denote the levels of the potential and the electric field strength.

\subsection{Analysis of Dynamic sensitivity}

Generally, the ring electrode has to be connected to a preamplifier to enhance its loading capability and amplify the signal. Thus the frequency characteristics of measurement system are determined by the characteristics of both the electrode and the conditioning circuit.

The "dynamic" electrostatic sensors detect the variation of the charge induced on the electrode. Approximately, the function of the conditioning circuit can be expressed as differentiation, i.e. the output voltage of the conditioning circuit is proportional to the rate of change of the induced charge, 


$$
U_{o}(t) \propto \frac{{ }^{\jmath} Q_{i}(t)}{d t}
$$

where $U_{o}(t)$ is the output of the conditioning circuit, $Q_{i}$ is the induced charge on the electrode, $\propto$ stands for "proportional to".

It can be seen that, for a given circuit, voltage $U_{\mathrm{o}}(t)$ varies with $d Q_{i} / d t$, which depends on the location, radial and axial velocities of the moving charged micro-column element. The following derivation is to establish $Q_{i}$ and $d Q_{i} / d t$ as the function of the location $(x, r, \varphi)$ where a micro-column element with unity charge travels at the radial velocity $V_{r}$, axial velocity $V_{x}$ and produces $Q_{i}$ and $d Q_{i} / d t$. Hereafter $Q_{i}$ is expressed as $Q_{i}(x, r, \varphi)$ to emphasize its location dependency.

Due to the sensor's symmetrical axial geometry, the change in angular position of the charged element does not affect the induced charge on the ring-shaped electrode. Hence $Q_{i}(x, r, \varphi)=Q_{i}(x, r)$. The simulation can be implemented in the rectangular region as depicted in Fig. 6 . The origin $(x=0, r=0)$ is at the geometric center of the sensor.

For given radial positions, for example $r=0$ and $80 \mathrm{~mm}$ for a sensor with the diameter of 14 " $(355.6 \mathrm{~mm})$ and the electrode width of $2 \mathrm{~mm}$, the induced charge, $Q_{i}(x, r)$ by the micro-column element of unit charge originated from the location $(x=0, r=$ constant $)$ decreases with its axial location $x$ when this micro-column element moves in parallel with the pipe central line towards either up or down stream direction. This can be seen in Fig. 7(a). Whilst for a given axial position, e.g. $x=0$ and $\mathrm{x}=50 \mathrm{~mm}, Q_{i}(x, r)$ increases monotonously with $r$, but it decreases with $r$ when the element approaching the edge of the sensing volume shown in Fig. 7(b).

The boundary of the sensing volume in this paper is defined as the edge where $Q_{i}$ has dropped to the value less than $1 \%$ of the maximum, the sensing volume under the above definition is shown in Fig. 8 where the belt represents the ring probe and the ellipsoidal body is the sensing volume. It is clear though that the sensing volume under this definition is much larger than the geometric volume covered by the electrode.

The following analytical expression via curve-fitting is derived based on the data contained in Fig. 7(a). It can be seen that a double Gaussian model offers high fitting accuracy.

$$
Q_{i}(x, r)=1(r) e^{-3(r) x^{2}}+\zeta(r) e^{-\gamma(r) x^{2}}
$$

where $A, B, C, D$ are functions of $r$, the radial coordinate of the element for a given electrode.

Fig.9 illustrates the variation of $Q_{i}(x, r)$ with $x$ at $r=80$, the analytical equation at this radial location is for the sensor with the dimensions given above.

$$
\left.Q_{i}(x, r)\right|_{r=0}=1.2725 e^{-13.793 x^{2}}+1.0953 e^{-/ 11.63 x^{2}}
$$

Fig. 10 shows a charged element positioned at $(x, r, \varphi)$, and the projections of its velocity vector $V_{\varphi}, V_{x}$, and $V_{r}$ on the angular, axial, radical coordinates respectively. When a charged micro-column travels along this circumference where this element situates on, the induced charge on the ring probe won't change due to the symmetrical configuration of the sensor. Thus the velocity $V_{\varphi}$ does not contribute to the variation of the total induced charge; only $V_{x}$ and $V_{r}$ need to be considered.

Defining the dynamic sensitivity $S_{\text {dynamic }}$ as the ratio between the derivative of induced charge to time on the probe and the unit positive charge, when the micro-column carrying unit coulomb of charges, moves at a velocity in the sensing volume, i.e.

$$
S_{\text {dynamic }}=\frac{1 Q_{i}(x, r, \varphi}{d t} / 1
$$

$S_{\text {dynamic }}$ has an unit of $\mathrm{s}^{-1}$. Substituting $Q_{i}(x, r, \varphi)$ in Equation (12) with the right side of Equation (14), we have,

$$
\begin{aligned}
& \left.S_{\text {dynamic }}\left(x, r, V_{x}, V_{r}\right)=-x\left(B(r) A(r) e^{-3(r) x^{2}}+\right)(r) C(r) e^{-)(r) x^{2}}\right) \cdot V_{x}+A^{\prime}(r) \cdot e^{-3(r) x^{2}} \\
& \left.+r^{\prime}(r) \cdot e^{-)(r) x^{2}}-1(r) x^{2} B^{\prime}(r) \cdot e^{-3(r) x^{2}}-\gamma(r) x^{2} D^{\prime}(r) \cdot e^{-)(r) x^{2}}\right) V_{r}
\end{aligned}
$$


It is clear from equation (15) that the dynamic sensitivity is dependant on the spatial position $(x, r)$, axial velocity $V_{x}$ and radial velocity $V_{r}$ of the charged element.

As an example, if this micro-column carrying the unit charge travels along the pipe line at $r=80 \mathrm{~mm}$, the induced charge on the electrode of $355.6 \mathrm{~mm}$ diameter and $2 \mathrm{~mm}$ width, i.e. the dynamic sensitivity will be,

$$
\left.S_{\text {dynamic }}\left(x, r, V_{x}, V_{r}\right)\right|_{r=80 m m, V_{r}=0}=\frac{t Q_{1}(x, r)}{d t}=\left[-0.1437 e^{-1 / 3.793 x^{2}}-0.0164 e^{-1 / 11.63 x^{2}}\right] \cdot x \cdot V_{x}
$$

This is a typical case of equation (15) when ignoring the radial velocity $V_{r}$ as shown in Fig.11. .

The effect of radial velocity on the sensitivity can be analyzed in the same way.

According to the definition of the sensitivity in this paper, the output signal of the conditioning circuit is proportional to $d Q_{r} / d t$ when the charged particle stream passes through the sensing zone at radius $r$. For simplicity, assume the proportion factor is unity, the output of the conditioning circuit is

$$
\frac{d Q_{r}}{d t}=\sum_{(x, r, \varphi \equiv} q x, r, \varphi \cdot S_{\text {dynamic }}\left(x, r, V_{x}, V_{r}\right)
$$

where $q(x, r, \varphi)$ is the charged local element and $S_{\text {dynamic }}\left(x, r, V_{x}, V_{x}\right)$ is the sensitivity, $\Omega$ denotes the sensing volume depicted in Fig. 9, $Q_{r}$ is the total charge induced on the entire ring probe.

\subsection{Experiment and analysis}

Fig. 12 shows a diagram of the belt rig in the laboratory of the Teesside University, which is used to validate the theoretical analysis discussed above. The rubber belt is pulled by two pulleys, one of which is driven by a DC motor. The speed of DC motor is adjustable to set the belt velocity from $5 \mathrm{~m} / \mathrm{s}$ to $25 \mathrm{~m} / \mathrm{s}$. A brush is placed at one side to generate electrical charges. The charged belt is used to simulate a stream of charged micro-columns or charged particles traveling along axial direction. The diameter of the sensor is 14 " $(355.6 \mathrm{~mm})$, and the width of the electrode is $2 \mathrm{~mm}$. These data are used for the numerical simulation in Section 1.3.

Fig.13 (a) shows the relationship between the belt velocity (V) and the root mean square value, $r m s$ of the sensor output at $\mathrm{r}=80 \mathrm{~mm}$, the vertical coordinate is the normalized value of the output root mean square value. It can be seen that the value of $r m s$ increases with belt conveyance velocity. Fig. 13 (b) depicts the rms versus d, the distance of the belt to the pipe wall at $\mathrm{V}_{\mathrm{x}}=17 \mathrm{~m} / \mathrm{s}$. It is clear the signal level decreases with $\mathrm{d}$.

\subsection{Discussions}

There may be three reasons for the signal increasing with the belt velocity. At first, higher velocity means more intensive friction between the belt and the brush, and between the belt and air, which produces more charges; secondly, even the charge carried by the belt unchanged, the sensitivity increases with axial velocity $\mathrm{V}_{\mathrm{x}}$, it can be seen from Equation (16) and (17); in addition, if the radial movement of the belt represented by $\mathrm{V}_{\mathrm{r}}$ is taken into account, the contribution from it may be also important.

It was observed that the belt vibration was more significant in the radial direction with the increased speed of the motor for this particular belt rig. The standard deviation to mean value ratio of rms was significantly higher than at lower velocity.

In order to analyze the cause of the signal at the output of the meter under variable belt velocity, we replace the dynamic sensitivity in equation (17) with that in equation (15). It follows,

$$
\begin{aligned}
& \frac{d Q}{d t}=\sum_{(x, r, \varphi \equiv}-q\left(x, r, \varphi \cdot x\left(B(r) A(r) e^{-3(r) x^{2}}+\right)(r) C(r) e^{-)(r) x^{2}}\right) \cdot V_{x}+\left(x, r, \varphi \cdot\left(A^{\prime}(r) \cdot e^{-i(r) x^{2}}\right.\right. \\
& \left.\left.+r^{\prime}(r) \cdot e^{-)(r) x^{2}}-1(r) x^{2} B^{\prime}(r) \cdot e^{-3(r) x^{2}}-Y(r) x^{2} D^{\prime}(r) \cdot e^{-)(r) x^{2}}\right) V_{r}\right\}
\end{aligned}
$$


It can be seen that $\mathrm{dQ} / \mathrm{dt}$ depends on $\mathrm{q}(\mathrm{x}, \mathrm{r}, \varphi)$ and its distribution, belt velocity in $\mathrm{x}$ and $\mathrm{r}$ directions.

Here presented is an interesting case in which the charges on the belt are assumed to be uniformly distributed over $-\leq \leq \leq$, where $-l$ and $l$ are the boundaries of the sensing volume along a given flow line, from Fig.11, it can be seen that the sum of the terms associated with $V_{x}$ in (18) approaches zero. Equation (18) can then be reduced into:

$$
\begin{aligned}
& \frac{d Q}{d t}=\sum_{(x, r, \varphi \equiv} q\left(x, r, \varphi \cdot\left(A^{\prime}(r) \cdot e^{-B(r) x^{2}}+C^{\prime}(r) \cdot e^{-D(r) x^{2}}-A(r) x^{2} B^{\prime}(r) \cdot e^{-B(r) x^{2}}-C(r) x^{2} D^{\prime}(r) \cdot e^{-D(r) x^{2}}\right) V_{r}\right. \\
& =\sum_{(x, r, \varphi \equiv} q\left(x, r, \varphi K(r, x) V_{r}\right.
\end{aligned}
$$

where $K(r, x)=1^{\prime}(r) \cdot e^{-3(r) x^{2}}+\gamma^{\prime \prime}(r) \cdot e^{-\jmath(r) x^{2}}-1(r) x^{2} B^{\prime}(r) \cdot e^{-3(r) x^{2}}-\gamma(r) x^{2} D^{\prime}(r) \cdot e^{-\jmath(r) x^{2}}$ which is independent of $q(x, r, \varphi)$ and $V_{r}$. In this sense, the output signal of the sensor is related to the radial velocity and the amount of charge carried by the moving belt. The velocity $\mathrm{V}_{\mathrm{x}}$ does not affect the signal.

It can not be verified in reality if the charges were uniformly distributed along the belt or not. However, for the rig with a belt of uniform property it is reasonable to imagine that there could be more contribution from the radial movement to the sensor's output than that from the movement along the pipe axis.

In pneumatic conveying system, it has also been observed that at high velocity, the air solids flow is more turbulent, and the signal rms value is higher. This is due to the fact that there is intensified solids radial movement. This may also confirm that the effect of the radial movement of the solids is dominant.

\subsection{Conclusions}

In this paper, the 3-D finite element method (FEM) is employed to investigate the dynamic sensitivity of the electrostatic sensor. Theoretical analyses reveal that the dynamic sensitivity depends on the spatial location, the axial and radial velocities of the charged element. From experimental results using a belt rig, it confirmed the theoretical analysis regarding the relation between sensitivity and axial velocity as well as the relation between sensitivity and flow stream spatial position. This is the first attempt to identify the effect of the radial velocity on the output signal of the dynamic electrostatic sensor. The test results seemed to support the theoretical analysis in this aspect too. However further in depth investigation is required and a new rig to create radial motion only may be necessary for such investigation.

\section{Acknowledgement}

This work was funded by European Research Fund for Coal and Steel, the contract number RFC-CR-03005. The authors also would like to express their gratitude to the Science Foundation of Ministry of Education of China for the New Teacher (No. 20090092120064), to the National Natural Science Foundation for youths (No. 50906013).

\section{Reference:}

1 A.G.Bailey, Charging of solids and powders, J. Electrostatics, 30 (1993) 167-180.

2 J. Guardiola, V. Rojo and G Ramos, Influence of particle size, fluidization velocity and relative humidity on fluidized bed electrostatics, J. Electrostatics, 37 (1996) 1-20.

3 D.I.Armour-Chelu and S.R.Woodhead, Comparison of the electric charging properties of particulate materials in gas-solids flows in pipelines, J. Electrostatics 56 (2002) 87-101.

4 D.K.Donald, Electrostatic contribution to powder-particle adhesion, J. Applied physics, 40-7 (1969) 3013-3019.

$5 \mathrm{~S}$. Matsusaka, M. Oki and H. Masuda, Bipolar charge distribution of a mixture of particles with different electrostatic characteristics in gas-solids pipe flow, Powder Technology, 135\&136 (2003) 150-155.

6 M.Nifuku and T.Sasaki, Static electrification phenomena in pneumatic transportation of coal, J. Electrostatics, 23 (1989) 45-54.

7 T.B.Jones and J,L,King, Powder Handling and Electrostatics-Understanding and Preventing Hazards, Lewis, Publishers INC., Chelsea, Michigan (1991). 
8 S.Kanazawa, Submicron particle agglomeration and precipitation by using a bipolar charging method, Journal of Electrostatics, 93 (1992) 193-209.

9 D.K.Yanar and B.A.Kwetkus, Electrostatic separation of polymer powders. J. Electrostatics, 35 (1995) 257-266. 10 W.Kleber and B.Makin, Triboelectric powder coating: a practical approach for industrial use, Part. Sci. Technol. 16 (1998) 43-53.

11 L. Reyderman and S. Stavchansky, Electrostatic spraying and its use in drug delivery-cholesterol micro-spheres, International Journal of Pharmaceutics, 124(1) (1995)75-85.

12 J.Ma and Y.Yan, Design and evaluation of electrostatic sensors for the measurement of velocity of pneumatically conveyed solids, Flow Measurement and Instrumentation 11(2000) 195-204.

13 Y. Yan, L. Xu and P. Lee, Mass Flow Measurement of Fine Particles in a Pneumatic Suspension using Electrostatic Sensing and Neural Network Techniques, IEEE Trans. Instrumentation and Measurement, Vol 55 No. 6 (2006) 2330-2334.

14 J. Q. Zhang and Y. Yan, On-line continuous measurement of particle size using electrostatic sensors, Powder Technology 135-136(2003) 164-168.

15 L. Peng; Y. Zhang and Y. Yan, Characterization of electrostatic sensors for flow measurement of particulate solids in square-shaped pneumatic conveying pipelines, Sensors \& Actuators: A. Physical, Vol 141 No. 1 (2008) 59-67.

16 J. Zhang and J. Coulthard, Theoretical and experimental studies of the spatial sensitivity of an electrostatic pulverized fuel meter, J. Electrostatics 63 (2005) 1133-1149.

Fig. 6 Simulation region


Fig.10 Projections of velocity

Fig.9 Double Gaussian curve fitting

Fig.11 Dynamic sensitivity variation along a flow line $(\mathrm{r}=80 \mathrm{~mm})$

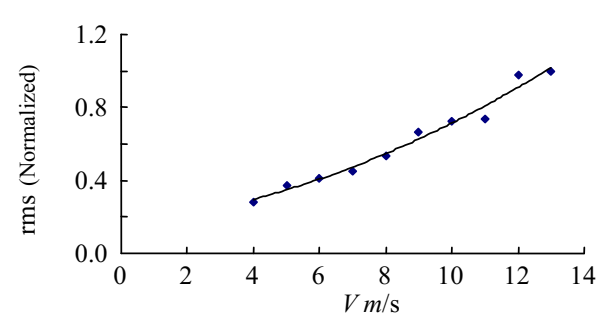

(a)

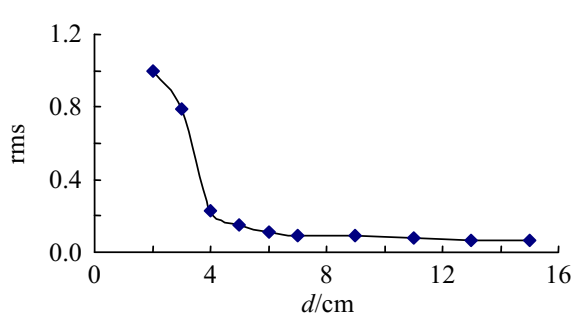

(b)

Fig.13 Belt velocity and signal root mean square value 